\title{
CanMEDS is a theory
}

\author{
Rachel Ellaway ${ }^{1}$
}

Received: 11 October 2016/Accepted: 13 October 2016/Published online: 18 November 2016

(C) Springer Science+Business Media Dordrecht 2016

It can be hard to escape the influence of competency frameworks in medical education today, assuming of course that escape is what is desired or required. In Canada the dominant competency framework is 'CanMEDS', a model of an ideal physician structured around seven top-level roles of a physician (medical expert, professional, communicator, collaborator, scholar, leader, and health advocate) along with associated sub-competencies and milestones (see canmeds.royalcollege.ca/en/framework). Of course, CanMEDS is not the only framework in use in contemporary medical education; others have been developed in and for different contexts, such as the framework from the ACGME in the United States and the eponymous Scottish Doctor. However, CanMEDS seems to be the one with the widest uptake (Norman et al. 2014) so I will use it as an exemplar of competency frameworks in general. My focus in this editorial is not on championing or criticizing competency frameworks (full declaration: I was a minor contributor to the latest edition of CanMEDS) but rather to cast these models and the thinking they reflect in a particular academic light. I will frame this in the form of a simple proposition: 'CanMEDS is a theory'. Let me elaborate.

Although there are variations in terms of what their essential elements are and how they are related (or not), competency frameworks set out, whether explicitly or implicitly, a vision for the ideal physician. The common underlying proposition is that the closer a trainee or practicing physician is to reflecting all aspects of the framework, the more ideal a practitioner they are. Conversely, the more inconsistencies there are between a trainee and the ideal, the more that trainee is considered to be failing to develop or perform as expected. Clearly the application of this proposition informs the expectations and the applications of these frameworks.

A theory is "a set of interrelated constructs, definitions and propositions that presents a systematic view of phenomena by specifying relations among variables, with the purpose of explaining and predicting the phenomena" (Kerlinger 1986). I would argue that

Rachel Ellaway

rachel.ellaway@ucalgary.ca

1 University of Calgary, Calgary, Alberta, Canada 
CanMEDS and the many other competency frameworks currently in circulation are theories according to these criteria; they outline a series of propositions and relationships that collectively define an ideal. The primary utility of these models is in comparing individuals to the ideal with purpose of identifying how well they do or do not reflect the ideal and thereby to explain and, to an extent, to predict future performance, developmental needs, and other phenomena. I would therefore argue that CanMEDS is a theory, even if it is not expressed in ways that are immediately recognizable as such.

There are many implications that flow from this argument. From a scholarly perspective perhaps the most important is the principle of provisionality (Kuhn 1962). This does not mean that we should reject these (or any other) theories outright but rather that we should be clear that, while these theories can be used a priori to structure inquiry, they can and should be rigorously tested and challenged. I would therefore argue that it is problematic if we use CanMEDS (or any other competency framework) without framing it as a theory. Doing so acknowledges our responsibilities as scientists to take a critical stance towards any theory on which we hang our work (Popper 1963).

A critical stance may involve taking one or more critical perspectives. For instance, we might test or challenge the form of a framework by considering the presence and absence of elements and relationships between elements (Whitehead et al. 2014). We might explore whether all elements need to be fully met or whether a good physician be strong in some areas but less so in others. Indeed, we might want to focus on establishing minimum thresholds that are acceptable for individual elements and in various combinations. This suggests that we should also explore whether and in what ways elements are independent or interdependent of each other.

We might wish to focus on the concept of defining an ideal physician against which all others are judged, and in doing so, we might consider their conceptual inconsistencies. For example, the assertion of defined roles is a deontological position (an assumption that these characteristics are in and of themselves essential) while their connections to competency is a consequentialist position (an assumption that the value of these roles is derived from the outcomes of embodying them in training programs); can they meaningfully be both? Another consequentialist stance would be to explore how effective the tools and activities that use the framework are, what the affordances of the framework are, and what constraints the framework has on practice and on thinking about practice (educational, medical, and scholarly).

We might, on the other hand, explore the provenance of these frameworks; how they were developed and how they reflect the times and cultures from which they sprang. Moreover, as with any innovation, while a framework may address those problems it was designed to target, it is likely to miss or even create other problems that its designers, with the greatest foresight and good will, did not foresee (Norman et al. 2014). We might also consider how generalizable a framework is. For instance, why do we not have a single global competency framework for physicians? One answer is that it would seem that, even where there is a broad consensus over what characteristics a physician should have, the specifics in terms of scope, priority, and fit to local healthcare systems can vary according to context (Ringsted et al. 2006).

There may also be utility in exploring how frameworks can be and are used as tools of consensus, coordination, or control. Finally, returning to implementation issues, we may wish to consider how individuals and collectives react to the use of these frameworks or we might consider the ways in which they are adapted or reconfigured to suit specific needs and contexts (Varpio et al. 2012). Clearly, each critical perspective (and I don't claim that 
this is an exhaustive list) affords different frames of inquiry and has different implications and utility in advancing health sciences education.

Competency frameworks may have great practical value in terms of creating a consensus standard, a common reference point, and a system-wide axis of organization. But they are also works in progress; CanMEDS, as with any theory, has utility within the paradigms it sprang from but these will change over time. This is not simply a matter of acknowledging the underlying theories for these frameworks (van der Vleuten et al. 2010); it is a matter of responding to the propositional and provisional nature of frameworks by treating them as theories, with all that implies. After all, by organizing systems of medical education around these frameworks, we send bold statements about health professional education that should be robustly tested. We need to consider all of our frameworks as theories and their many applications need to be evaluated critically, rigorously, and objectively on that basis. It is only by doing so that we move our field forward.

\section{References}

Kerlinger, F. N. (1986). Foundations of behavioral research (3rd ed.). San Diego, CA: Harcourt Brace.

Kuhn, T. S. (1962). The structure of scientific revolutions. Chicago, USA: The University of Chicago Press. Norman, G., Norcini, J., \& Bordage, G. (2014). Competency-based education: Milestones or millstones? Journal of Graduate Medical Education, 6(1), 1-6.

Popper, K. (1963). Conjectures and refutations: The growth of scientific knowledge. London, UK: Routledge.

Ringsted, C., Hansen, T. L., Davis, D., \& Scherpbier, A. (2006). Are some of the challenging aspects of the CanMEDS roles valid outside Canada? Medical Education, 40(8), 807-815.

van der Vleuten, C. P., Schuwirth, L. W., Scheele, F., Driessen, E. W., \& Hodges, B. (2010). The assessment of professional competence: Building blocks for theory development. Best Practice and Research. Clinical Obstetrics and Gynaecology, 24(6), 703-719.

Varpio, L., Bell, R., Hollingworth, G., Jalali, A., Haidet, P., Levine, R., et al. (2012). Is transferring an educational innovation actually a process of transformation? Advances in Health Sciences Education: Theory and Practice, 17(3), 357-367.

Whitehead, C., Selleger, V., van de Kreeke, J., \& Hodges, B. (2014). The 'missing person' in roles-based competency models: A historical, cross-national, contrastive case study. Medical Education, 48(8), $785-795$. 